\title{
Surgical and Clinical Confirmation of Temporal Bone CT Findings in Patients with Otosclerosis with Failed Stapes Surgery
}

\author{
J. Whetstone, A. Nguyen, A. Nguyen-Huynh, and B.E. Hamilton
}

\begin{abstract}
BACKGROUND AND PURPOSE: Prior descriptions of imaging after failed stapes procedures for otosclerosis predated currently available CT technology and/or failed to assess commonly used metallic implants. The purpose of this study was to correlate temporal bone CT findings with clinically and intraoperatively determined causes of surgical failure.
\end{abstract}

MATERIALS AND METHODS: All patients with otosclerosis undergoing stapedectomy between December 1999 and December 2010 were identified from a search of neurotology clinical records. Patients presenting because of failed stapes surgery and having temporal bone CT scans at the time of revision surgery or clinical evaluation were included. Imaging and clinical records were retrospectively evaluated by a medical student, radiology resident, and senior neuroradiologist. Stapes prosthesis complications and relevant anatomic CT findings were correlated to clinical and intraoperative findings.

RESULTS: Twenty-two of 340 patients met inclusion criteria. Temporal bone CT findings were correlated to intraoperative findings in 17 of 22 patients and to clinical findings in 5 of 22 patients. Surgically confirmed abnormalities included 7 of 7 incus erosions, 3 of 6 piston re-sizings, 3 of 5 granulation tissues, 3 of 5 prosthesis disconnections, 3 of 4 obliterative otosclerosis, 2 of 2 oval window dislocations, and 1 labyrinthine ossificans. Clinically confirmed abnormalities included 2 cases each of superior semicircular canal dehiscence, and wrong piston size, and 1 each of piston disconnection, labyrinthine ossificans, and intravestibular footplate.

CONCLUSIONS: CT evaluation in the setting of failed stapes surgery is challenging. Many postoperative complications such as piston migration, incus necrosis, and overt vestibular penetration are well recognized on temporal bone CT. Of particular note, superior semicircular canal dehiscence is an important contraindication to stapes surgery.

ABBREVIATIONS: $\mathrm{CHL}=$ conductive hearing loss; $\mathrm{SNHL}=$ sensorineural hearing loss; $\mathrm{MHL}=$ mixed hearing loss; $\mathrm{SSCD}=$ superior semicircular canal dehiscence; $\mathrm{OW}=$ oval window OtoO $=$ obliterative otosclerosis

O tosclerosis affects approximately $1 \%$ of the population (more common in whites but rare in African Americans, Asians, and Native Americans) and usually presents early as conductive hearing loss (CHL) and later as mixed CHL and sensorineural hearing loss (SNHL) in young adults. ${ }^{1-3}$ CHL occurs early on as the result of mechanical fixation of the stapes footplate within the oval window (OW) that prevents normal mobility. This limits transmission of energy as a fluid wave within the inner ear fluid and manifests as CHL. Surgical management of otosclerosis includes stapedectomy, which involves the creation of a small hole in the stapes footplate within the OW and removal of

Received September 2, 2013; accepted after revision October 22.

From the Departments of Radiology (J.W., A.N., B.E.H.) and Otolaryngology (A.N.-H.), Oregon Health and Science University, Portland, Oregon.

Please address correspondence to Bronwyn E. Hamilton, MD, Mail Code CR 135, 3181 SW Sam Jackson Park Rd, Portland, OR 97239; e-mail: hamiltob@ohsu.edu

http://dx.doi.org/10.3174/ajnr.A3829 portions or all of the stapes and implantation of a prosthesis to restore mechanical sound conduction.

Surgical treatment of CHL is evaluated postoperatively with 4 -frequency air-bone gap. Closure of the air-bone gap to $10 \mathrm{~dB}$ or less reflects surgical success and was achieved in $94.2 \%$ of patients in the largest prospective data base of stapedotomies for otosclerosis. ${ }^{4}$ If CHL persists after surgery, then revision surgery is usually indicated but is more challenging. ${ }^{5}$

High-resolution temporal bone CT is an important technique that can identify many causes of stapedectomy failure, particularly for recurrent or persistent CHL, and provides a roadmap for revision surgery. Although 2 prior excellent studies delineated many common causes of failed otosclerosis surgery, better CT technology and an improved understanding of other conditions contributing to hearing loss have intervened since their publication. ${ }^{6,7}$ One study, for example, addressed only fluoroplastic implants rather than the metallic implants that are typically used in the United States, ${ }^{6}$ whereas the other study is in part outdated 
Table 1: Temporal bone CT findings compared with intraoperative findings and clinical presentation in patients with revision stapedectomy

\begin{tabular}{|c|c|c|c|c|c|c|}
\hline Patient & CT Oto & Age, $y$ & Sex & Presentation & CT & Surgery \\
\hline 1 & B OtoF & 55 & $\mathrm{~F}$ & R MHL, vest & $\begin{array}{l}\text { R LPS with incus erosion, gas in } \\
\text { vestibule? }\end{array}$ & $\begin{array}{l}\text { R LPS with incus necrosis, fistula not } \\
\text { mentioned }\end{array}$ \\
\hline 2 & B neg & 44 & $\mathrm{~F}$ & R SNHL, vest & Neg & R long piston \\
\hline 3 & R OtoF & 35 & $\mathrm{~F}$ & L CHL, tinnitus & L prosthesis dislocation from OW & L long piston, loosening \\
\hline 4 & B OtoC & 68 & M & R MHL & $\begin{array}{l}\text { R LPS with disconnection, scar, } \\
\text { incus erosion, OW dislocation }\end{array}$ & $\begin{array}{l}\text { R LPS with disconnection, scar, incus necrosis, } \\
\text { OW dislocation }\end{array}$ \\
\hline 5 & ROtoF & 56 & $\mathrm{~F}$ & R SNHL, vest & $\mathrm{R}$ long piston & R long piston, incus medialized \\
\hline 6 & B OtoF & 80 & M & L SNHL & $\mathrm{L}$ long piston & $\begin{array}{l}\mathrm{LCl} \text { (piston length unconfirmed) } \\
\text { Clinical notes limited }\end{array}$ \\
\hline 7 & B OtoC & 56 & M & $\begin{array}{l}\text { L MHL, vest } \\
\text { R MHL }\end{array}$ & L LO, B OtoC, B OtoO & $\begin{array}{l}\text { L OtoO prior surgery } \\
\mathrm{R} \mathrm{Cl} \mathrm{limited} \mathrm{notes;} \mathrm{HL} \text { thought in part caused } \\
\text { by OtoC }\end{array}$ \\
\hline 8 & B neg & 44 & M & $\mathrm{LCHL}$ & L short piston & L short piston \\
\hline 9 & B OtoF & 61 & M & $\mathrm{R} \mathrm{CHL}$ & Neg & R OW dislocation, bone ingrowth \\
\hline 10 & B neg & 15 & $\mathrm{~F}$ & $\mathrm{RCHL}$ & R OW dislocation & R OW dislocation, scar, OtoO \\
\hline 11 & B OtoC & 51 & $\mathrm{~F}$ & R MHL & R OtoO & $\begin{array}{l}\text { R OtoO prior surgery } \\
\mathrm{R} \mathrm{Cl} ; \mathrm{HL} \text { probably caused by OtoC }\end{array}$ \\
\hline 12 & B neg & 54 & $\mathrm{~F}$ & $\mathrm{RCHL}$ & $\mathrm{R}$ incus erosion & R piston loose, incus necrosis, scar \\
\hline 13 & ROtoF & 30 & $\mathrm{~F}$ & $\mathrm{R} \mathrm{CHL}$ & $\mathrm{R}$ incus erosion & $\begin{array}{l}\text { R stapes not crimped and scarred to incus with } \\
\text { incus necrosis }\end{array}$ \\
\hline 14 & B OtoF & 31 & $\mathrm{~F}$ & $\mathrm{R} \mathrm{CHL}$ & $\begin{array}{l}\mathrm{R} \text { incus erosion and disconnection, } \\
\text { OtoO }\end{array}$ & $\mathrm{R}$ incus erosion and disconnection, OtoO \\
\hline 15 & ROtoF & 67 & $\mathrm{~F}$ & $\mathrm{R} C H L$, vest & Neg & R nonmobile piston surrounded by scar \\
\hline 16 & B neg & 53 & $\mathrm{~F}$ & $R$ vest & $\mathrm{R}$ long piston, incus erosion & $\mathrm{R}$ long piston, scar, incus necrosis \\
\hline 17 & ROtoF & 52 & $\mathrm{~F}$ & $L$ vest & $\begin{array}{l}\text { L incus erosion, prosthesis disconnection, } \\
\text { OW dislocation }\end{array}$ & $\begin{array}{l}\text { L piston medialized, disconnection, } \\
\text { incus necrosis }\end{array}$ \\
\hline
\end{tabular}

Note:-LPS indicates lateralized piston syndrome; CHL, conductive hearing loss; SNHL, sensorineural hearing loss; MHL, mixed hearing loss; R, right; L, left; B, bilateral; OtoF, fenestral otosclerosis; OtoC, cochlear otosclerosis; OtoO, obliterative otosclerosis; neg, negative; vest, vestibular symptoms; Cl, cochlear implantation; OW, oval window; LO, labyrinthine ossificans; HL, hearing loss.

because of technological advancements in CT technology and because anatomic considerations such as superior semicircular canal dehiscence (SSCD), labyrinthine ossificans, and obliterative otosclerosis (OtoO) were not recognized at that time as potential causes of surgical failure. ${ }^{7}$ The particular limitations of CT evaluation of metallic-type prostheses were also not described at that time. $^{7}$

We sought to perform a more contemporary evaluation of a series of consecutive patients with otosclerosis presenting for failed stapedectomy to better evaluate the potential role of preoperative temporal bone CT performed with the use of current technology.

\section{MATERIALS AND METHODS}

Patients undergoing stapes or stapes revision surgery at our institution between December 1999 and December 2010 were identified from a search of the department of neuro-otology clinical records. Patients presenting because of failed stapes surgery (those with new or persistent hearing and/or balance complaints after prior stapes surgery) and having temporal bone CT at the time of surgical or clinical evaluation were included in this retrospective review. Institutional review board approval was obtained with waiver of informed consent for this retrospective imaging and chart review. Temporal bone CT studies were reviewed in consensus in an unblinded fashion by a medical student, radiology resident, and neuroradiologist with a Certificate of Added Qualification in neuroradiology to identify imaging findings that could be correlated to surgical and clinical findings. The imaging and intraoperative reports were also discussed with a senior neurotologist.
Temporal bone CT protocol at our institution includes helically acquired 0.75 -mm-thick axial images on 1 of 2 16-section MDCT scanners from the top of the temporal bones through the tip of mastoids obtained without intravenous contrast. Images are routinely reconstructed in axial and coronal increments in bone and soft tissue algorithms. Postprocessing by use of oblique sagittal (Stenvers and Poschl views) type reformats and 3D volumerendered reconstructions were performed in selected cases when possible to better demonstrate abnormalities. High-quality multiplanar reformations and 3D reconstructions were not routinely possible in all cases in this retrospective study, given that the original dataset is not saved after a short period of time at our institution. Older studies in 4 cases included 1-mm-thick direct axial and coronal images by use of a single-section CT scanner.

CT images were reviewed to assess the location and position of the stapes prosthesis, appearance of the OW and round window, otic capsule attenuation, cochlea, vestibule, semicircular canals, ossicles, and tympanic cavity. Areas of abnormal soft tissue attenuation, abnormal bone formation, and ossicular fixation were assessed.

\section{RESULTS}

A total of 340 patients who had stapes surgery were initially identified from the department of neurotology clinical records. Sixty of $340(17.6 \%)$ had temporal bone CT examinations available in our digital PACS. A total of 22 of 60 (36.7\%) poststapedectomy patients ( 7 male, 15 female), ranging in age from 16-87 years (mean, 48 years) formed the basis of this review. Seventeen of these 22 patients had presurgical temporal bone CT studies that could be correlated with subsequent 

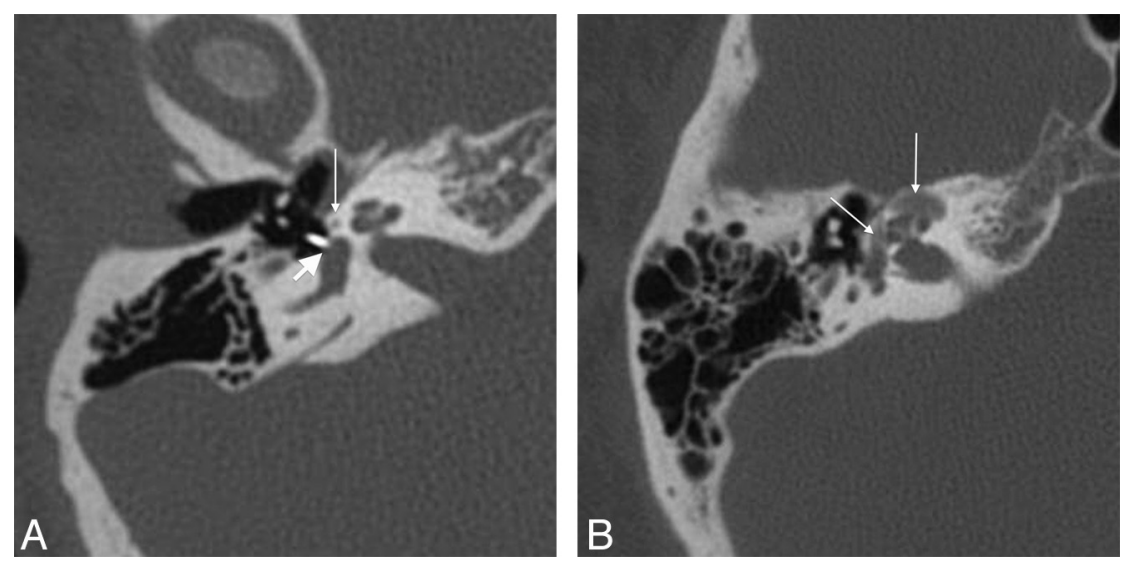

FIG 1. A, Axial CT in a 15-year-old girl with persistent conductive hearing loss poststapedectomy shows a small lucency in the left fissula antefenestrum (thin arrow), consistent with fenestral otosclerosis. Her piston prosthesis appeared short (thick arrow), without intravestibular penetration (surgically confirmed). $B$, Axial CT in a 56-year-old man with sensorineural hearing loss after stapedectomy shows extensive lucency surrounding the cochlea (arrows), consistent with cochlear otosclerosis.
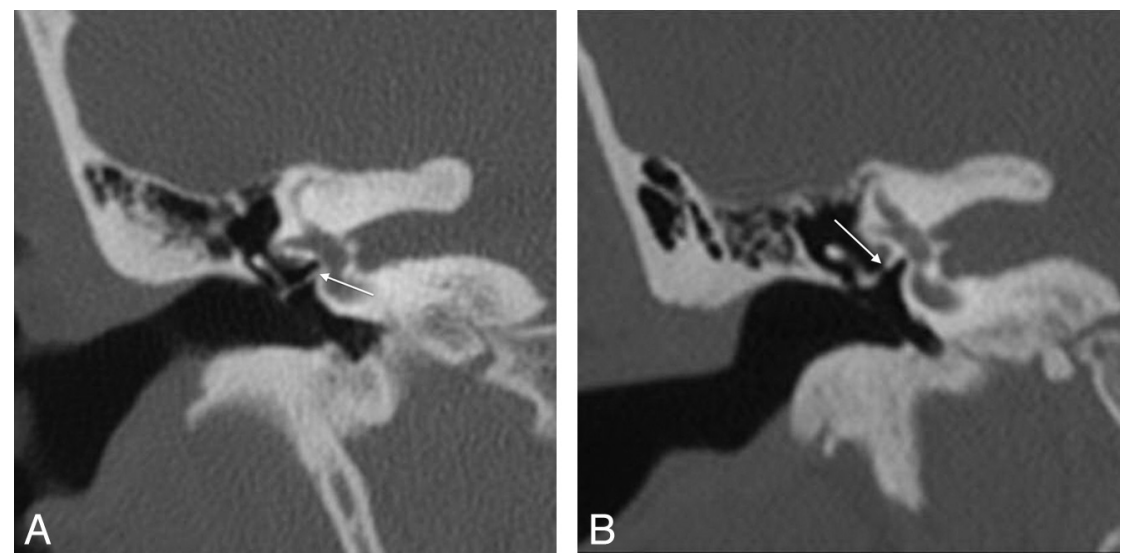

FIG 2. A, Coronal CT in a 40-year-old woman with recurrent right conductive hearing loss after stapedectomy shows inferior dislocation of the prosthesis (arrow) with respect to the oval window. B, Coronal CT in a 15-year-old girl with CHL shows superior dislocation of the stapes prosthesis from the OW (arrow).
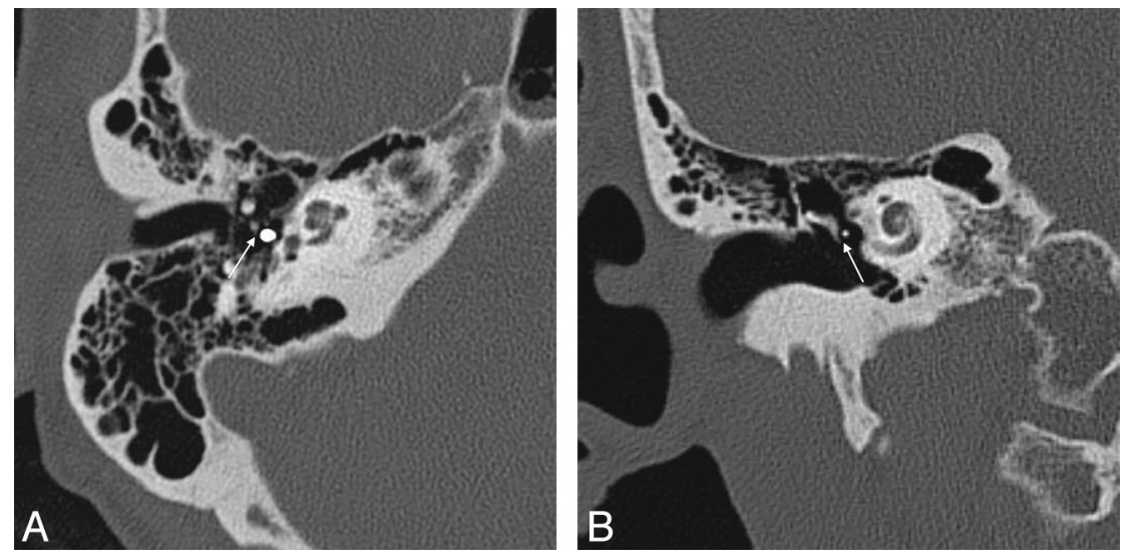

FIG 3. A, Axial CT in a 31-year-old woman with right conductive hearing loss after stapedectomy shows a gap (arrow) consistent with disconnected prosthesis. B, Coronal CT (same patient as in A) shows slender incus erosion and disconnection (arrow). This patient had intraoperatively confirmed disconnection and incus necrosis.

intraoperative findings. Fourteen of 17 had revision stapedectomies, whereas 3 of 17 had cochlear implant surgery in conjunction with prior stapes surgical reports plus clinical correlation. Five additional poststapedectomy patients ( 3 male, 2 female; mean age, 42 years) with 6 postoperative ears have not undergone revision surgery to date but had temporal bone CT studies that were reviewed and correlated to clinical follow-up only.

Persistent hearing or balance complaints in the 17 patients with stapedectomy that prompted revision stapes surgery in 14 and cochlear implantation in 3 included CHL in 8 patients, SNHL and/or vestibular symptoms in 5 patients, and mixed hearing loss (MHL) in 4 patients. The primary intraoperative findings correlated to clinical presentation in the 17 patients with revision stapedectomy are detailed in Table 1.

Otosclerosis was visible radiographically in 12 of $17(70.6 \%)$ of the surgical group and 3 of $5(60 \%)$ of the clinically confirmed group. Fenestral otosclerosis was visible at CT in 9 of 17 (52.9\%) of the surgical group and 3 of $5(60 \%)$ of the clinically confirmed group (Fig $1 A$ ). Cochlear otosclerosis was present in 3 of 17 $(17.6 \%)$ of the surgically confirmed and none of the clinically confirmed patients (Fig 1B).

\section{CT Findings in Surgically Confirmed Patients}

Preoperative temporal bone CT findings correlating to intraoperative findings included 2 of 2 cases of OW dislocation (Fig 2); 3 of 5 cases of prosthesis disconnection (Fig 3); 7 of 7 cases of incus erosion, 2 as part of the lateralized piston syndrome (Figs 4 and 5); 1 of 1 short piston (Fig 6); 2 of 5 long pistons (Fig 7); 3 of 5 with granulation tissue; and 3 of 4 surgically confirmed cases of OtoO (Fig 8). Three patients with no definite CT abnormality had the following intraoperative findings explaining surgical failure: 1 each of nonmobile piston encased in scar, piston dislocation from OW with new bone formation, and long piston. Additional incompletely seen CT findings at surgery included a case of prosthesis uncrimping with scar, though the CT did confirm incus erosion in this case that supported loosening.

CT findings identified but not mentioned in surgical notes included the following possible postoperative complications: 1 suspected long piston that received cochlear implant with limited intraoperative description, 1 piston 

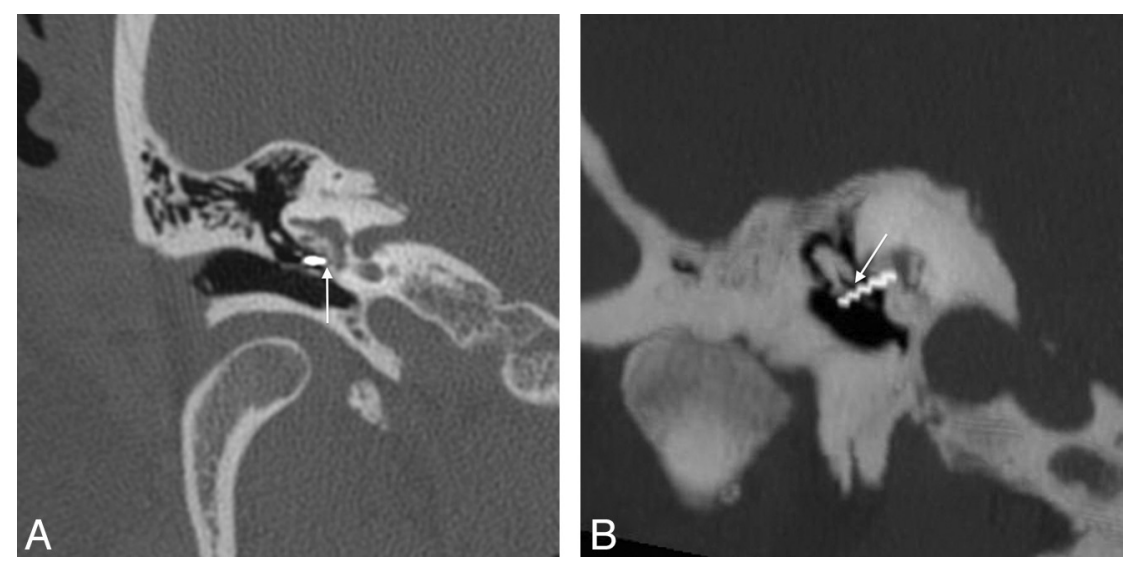

FIG 4. A, Axial CT in a 68-year-old man with recurrent mixed hearing loss shows findings suggesting the lateralized piston syndrome: prosthesis displacement inferior to the OW (arrow). Piston tip is encased in new otosclerotic bone. $B$, Coronal oblique multiplanar reformation (same patient as in A) shows incus erosion (arrow). Intraoperative findings confirmed scar tissue surrounding the stapes piston, causing prosthesis extrusion.
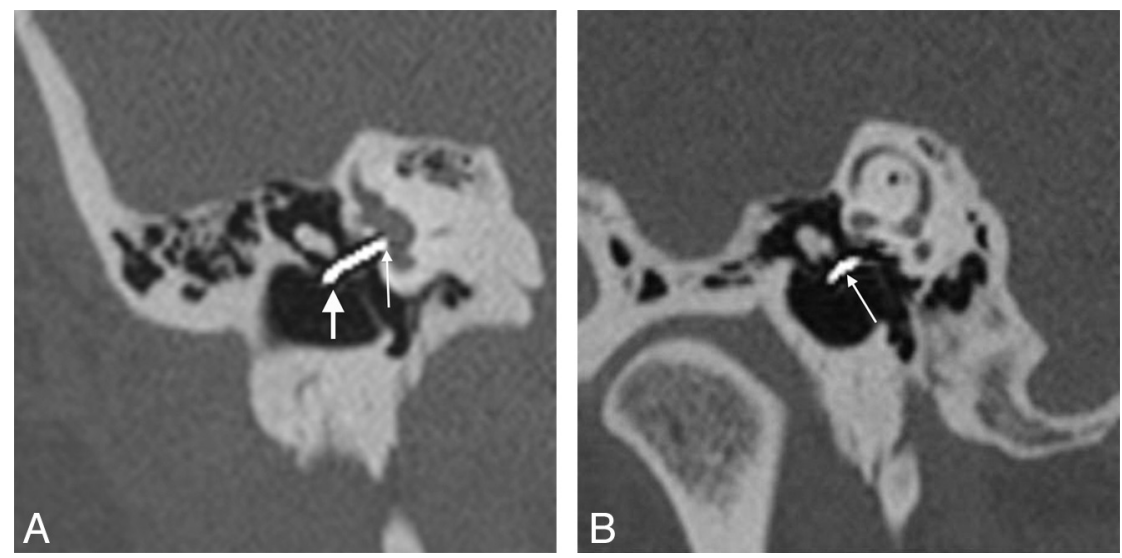

FIG 5. A, Stenver multiplanar reformation $\mathrm{CT}$ in a 55 -year-old woman with mixed hearing loss shows findings of lateralized piston syndrome. The piston is in the oval window, but no vestibular penetration (thin arrow) is noted. Note piston lateralization to the tympanic membrane (thick arrow). B, Poschl MPR (same patient as in A) shows the piston traversing the expected location of the incus long process, which is eroded (arrow).
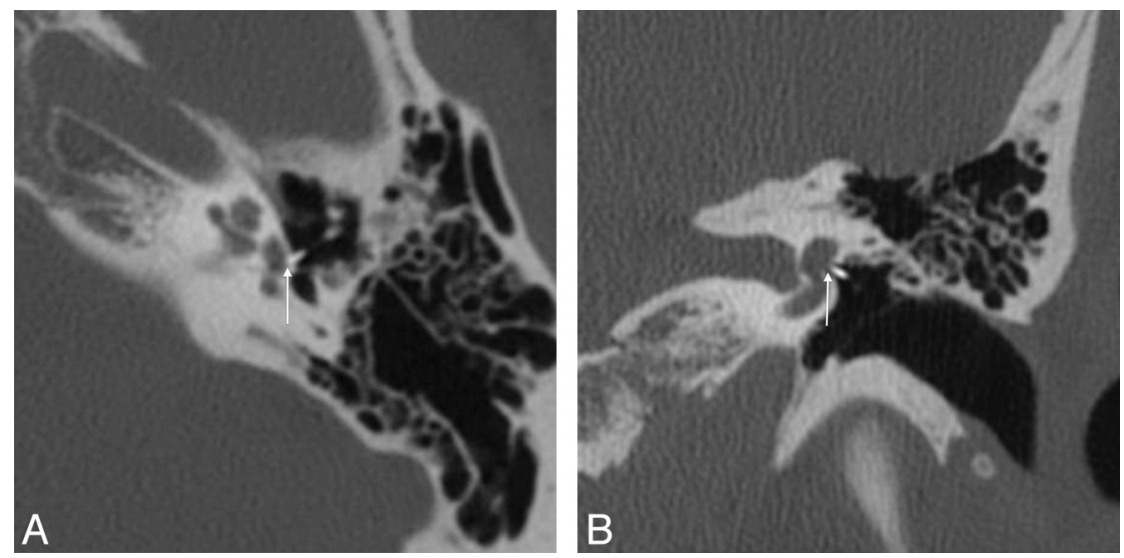

FIG 6. $A$, Axial $C T$ in a 44-year-old woman with conductive hearing loss demonstrates no vestibular penetration (arrow) by the piston prosthesis. B, Coronal CT multiplanar reformation in the same patient as in $A$ also shows no vestibular penetration (arrow), suggesting short piston. Intraoperative findings confirmed inadequate piston depth.

dislocation from the OW (in which concurrent incus erosion and prosthesis disconnection were seen and did correlate with CT), 1 possible intravestibular gas (in a patient with vestibular symptoms) without surgically confirmed labyrinthine fistula, and 1 prosthesis disconnection from the ossicular chain in which the only finding in this case at surgery was a suspected long piston, not evident at CT. Two additional CT anatomic findings were SSCD in a patient with ipsilateral CHL and labyrinthine ossificans in a patient with ipsilateral MHL that may have in part explained surgical failures.

\section{CT Findings in Patients with Clinical Confirmation Only}

The 5 patients evaluated having only clinical correlation showed abnormalities on temporal bone $\mathrm{CT}$ in 6 poststapedectomy ears (Table 2). One patient with postoperative SNHL had a long prosthesis with excessive vestibular penetration as a presumed cause. One patient with prior bilateral stapedectomies showed prosthesis disconnection and incus erosion correlating to MHL on 1 side and a "dead" ear (complete sensorineural deafness) on the contralateral side as the result of an intravestibular foreign body caused by footplate dislocation (Fig 9). Prosthesis dislocation from the OW and short piston probably explained $\mathrm{CHL}$ in 2 cases, respectively, whereas concurrent SSCD and labyrinthine ossificans could explain MHL in another (Fig 10). Interestingly, the patient with suspected short piston also had SSCD in addition to findings consistent with fenestral otosclerosis on her symptomatic but unoperated contralateral ear.

\section{DISCUSSION}

Otosclerosis is a genetically inherited osteodystrophy that affects the otic capsule of the temporal bone. Although autopsy series suggest a prevalence of $10 \%$, approximately $1 \%$ of the population is affected clinically. ${ }^{2}$ The inheritance pattern is autosomal dominant but with incomplete penetrance of $40 \%$ and variable clinical expression. ${ }^{8}$

Surgical treatment of otosclerosis has a high success rate, but revision stapedectomy is more challenging and prone to more surgical complications. Temporal bone CT is particularly useful for preoperative planning in such cases because it may reveal the suspected cause of surgical failure and/or may identify anatomic abnormalities that may alter the treatment plan.

Prior description of temporal bone CT in poststapedectomy patients was based on polytetrafluoroethylene implants in France ${ }^{6}$ that are not commonly used in the United States and on an 


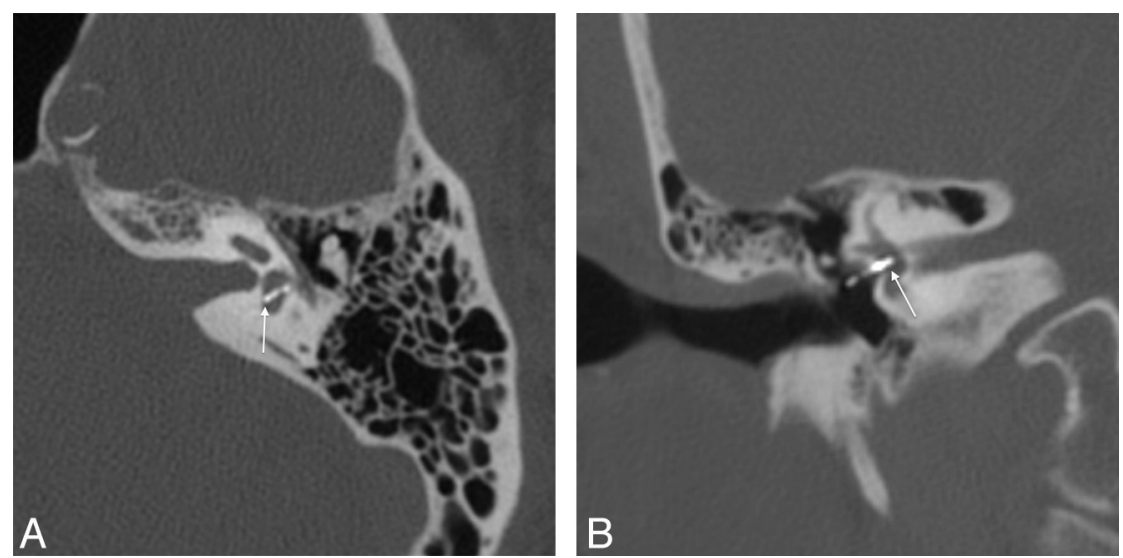

FIG 7. A, Axial CT in an 80-year-old man with sensorineural hearing loss shows deep intrusion into the vestibule (arrow). B, Coronal CT multiplanar reformation in a 68-year-old man with vestibular symptoms suggests deep intravestibular position of the stapes prosthesis (arrow). Long prosthesis and small labyrinthine fistula were confirmed intraoperatively.
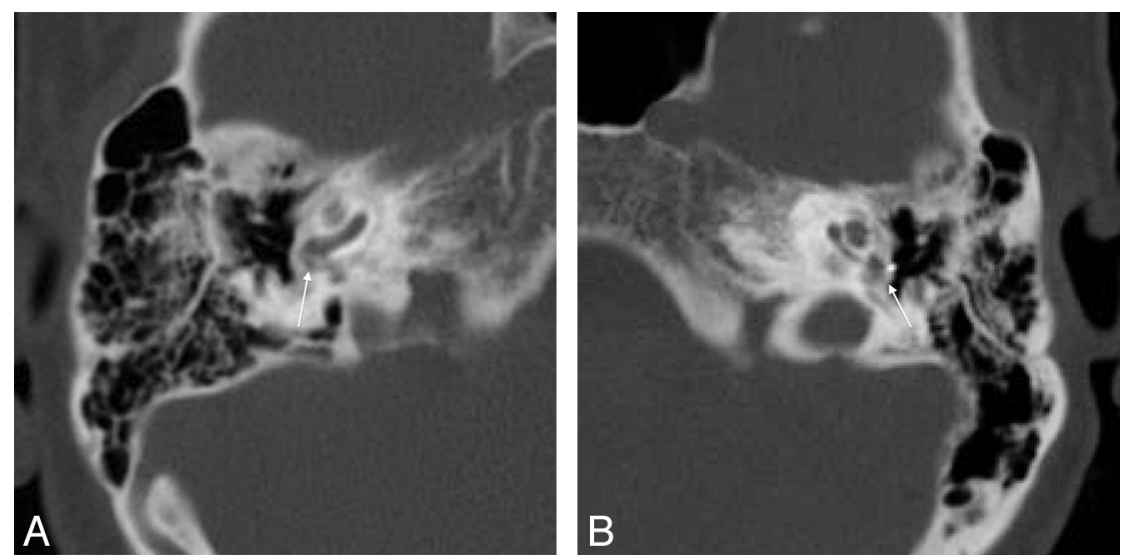

FIG 8. A, Axial CT in a 56-year-old man with persistent mixed hearing loss after stapedectomy demonstrates heaped-up lucent bone formation at the right round window consistent with obliterative otosclerosis (OtoO) (arrow). This patient also had probable superior semicircular canal dehiscence (not shown). B, Axial CT in the contralateral ear in the same patient as in $B$ also shows OtoO. Note tip of prior stapes piston embedded within otosclerotic new bone (arrow).

Table 2: Temporal bone CT findings in patients after stapedectomy with clinical confirmation only

\begin{tabular}{ccclll}
\hline Patient & Age, y & Sex & CT Oto & Presentation & \multicolumn{1}{c}{ CT Findings } \\
\hline 1 & 40 & F & B OtoF & R CHL, tinnitus & R prosthesis dislocation from OW \\
2 & 67 & M & B neg & R MHL & R SSCD, R LO \\
3 & 11 & F & B OtoF & R CHL & $\begin{array}{l}\text { R short piston } \\
\text { L SSCD (contralateral to operated ear) }\end{array}$ \\
4 & 41 & M & B neg & $\begin{array}{l}\text { B MHL } \\
\text { B MHL, vertigo }\end{array}$ & $\begin{array}{l}\text { L intravestibular foreign body (footplate) } \\
\text { R disconnection of prosthesis-incus, incus } \\
\text { erosion, OW dislocation } \\
5\end{array}$ \\
& 58 & M & B OtoF & L SNHL & L long piston \\
\hline
\end{tabular}

Note:- $\mathrm{CHL}$ indicates conductive hearing loss; SNHL, sensorineural hearing loss; $\mathrm{MHL}$, mixed hearing loss; OW, oval window; SSCD, superior semicircular canal dehiscence; LO, labyrinthine ossificans; R, right; L, left; B, bilateral; OtoF, fenestral otosclerosis; neg, negative.

older study from 1986 with the use of $1.5-\mathrm{mm}$-thick sections that predated MDCT. ${ }^{7}$ Many conditions such as SSCD are now only visualized because better spatial resolution and thinner section collimation is available with MDCT. ${ }^{9}$ MDCT further allows excellent MPR and 3D reformations that optimize detection of relevant pathology. ${ }^{10}$

Metallic stapes prostheses are more challenging to evaluate with CT because attenuation artifacts limit accurate evaluation of prosthesis size and intravestibular penetration. ${ }^{11-13}$ Although technique. most metallic prostheses were easy to evaluate in this study, a few were difficult to visualize, probably because of smaller prosthesis size and technical factors. It remains worthwhile commenting on suspected piston sizing abnormalities because most patients presenting with persistent CHL or other postoperative complications require surgical re-exploration, and preoperative CT findings improve surgical planning. Despite inaccurate size estimation on $\mathrm{CT}$, excessive vestibular penetration caused by migration or length seemed reasonable to suggest in this study if the piston tip depth exceeded $50 \%$ of the (normal) vestibule width.

Persistent or recurrent CHL after primary stapes surgery occurs in approximately $5.8 \%$ of cases, ${ }^{4}$ on the basis of residual air-bone gap $>10 \mathrm{~dB}$ after surgery. This most often results from prosthesis migration or dislocation $(>80 \%){ }^{12,14}$ The prosthesis may become disconnected or loose and may be evidenced by a gap in the ossicular chain-stapes structure. MPR and/or 3D reconstruction may best demonstrate the gap. The "lateralized piston syndrome" was seen in 2 of our cases, and it is characterized by lateral piston extrusion out of the OW that is often associated with incus necrosis. The piston itself may contact the tympanic membrane in more than half of cases, ${ }^{15}$ and was present in 1 of our cases.

Ossicular necrosis or resorption, evident by erosion on CT, may also lead to $\mathrm{CHL}$. The incus long process is most often involved because it is typically the point of prosthesis attachment. Erosion is potentiated by excessive piston length or insufficient crimping, which may transmit undue stress, though nonsurgical cases of incus erosion are also reported, thought to be related to ischemic resorption. ${ }^{16}$

Granulation tissue or new bone formation may result in recurrent CHL. Granulation tissue is seen as soft tissue attenuation, though small size, suboptimal technique, and/or motion may limit visualization. Intraoperatively identified functional immobility and prosthesis uncrimping were additional sources of false-negative CT that illustrate the fact that some complications may not be detectable with any anatomic imaging

Other findings that may contribute to poststapedectomy CHL are OtoO and ossicular fixation. OtoO reflects progression of otosclerosis and is seen as patchy lucent new bone formation. Bony 

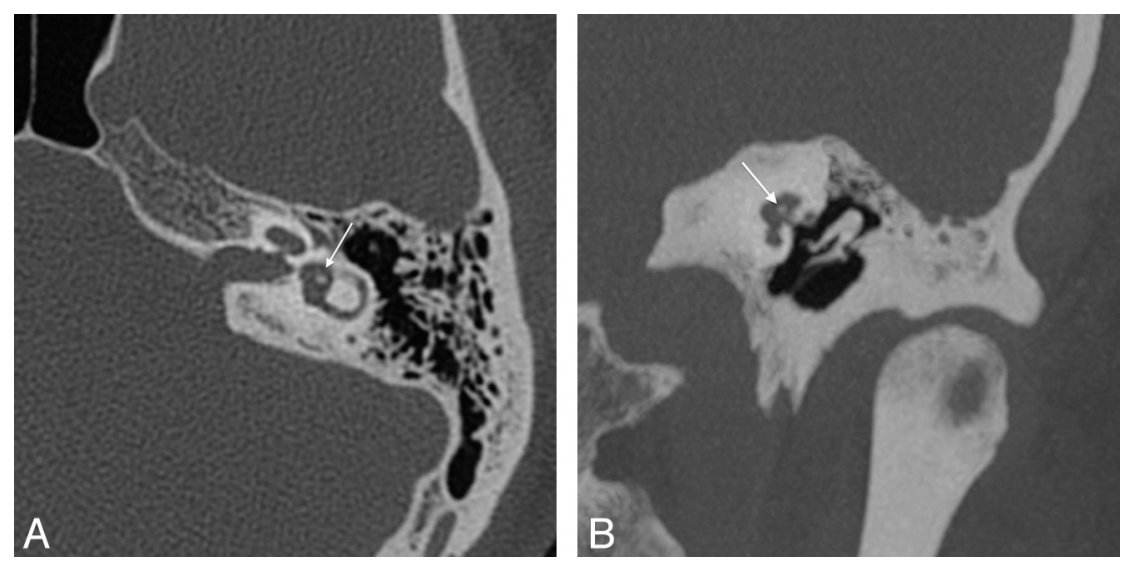

FIG 9. A, Axial CT in a 41-year-old man with vertigo and complete sensorineural hearing loss in the left ear after prior stapedectomy show focal hyperattenuation in the vestibule (arrow), consistent with intravestibular footplate dislocation. $B$, Coronal multiplanar reformation in the same patient as in $A$ shows intravestibular footplate dislocation (arrow).
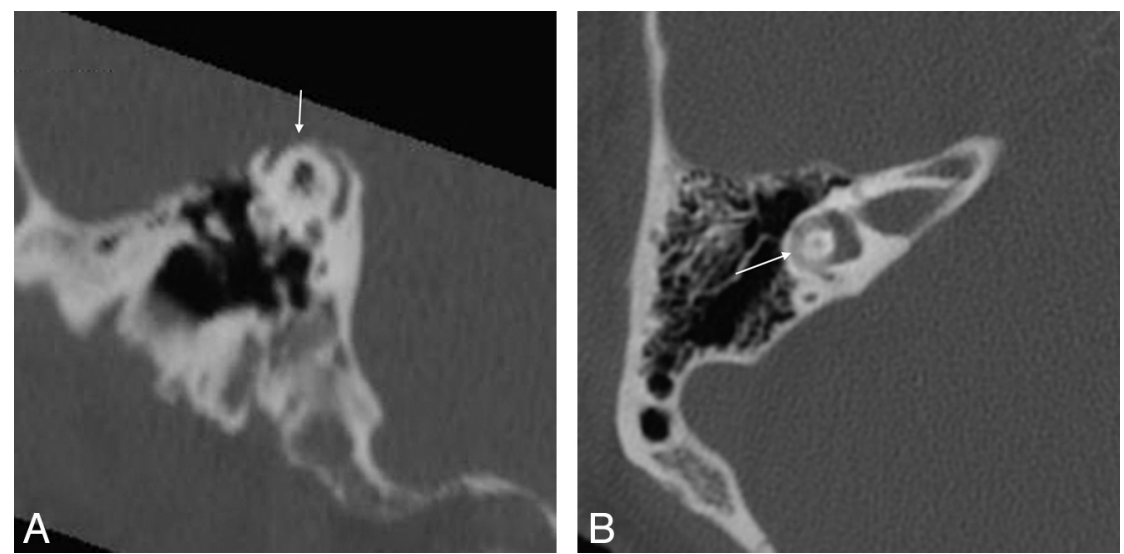

FIG 10. A, Poschl multiplanar reformation CT in a 67-year-old man with mixed hearing loss in the right ear after prior stapedectomy shows ipsilateral superior semicircular canal dehiscence (arrow) that probably explains surgical failure. B, Axial CT demonstrates ipsilateral labyrinthine ossificans (arrow) in the same patient as in $A$ that might also have contributed to his surgical failure.

round window or OW obliteration prevents normal mechanical conduction and affects surgical planning. Although not an absolute contraindication, surgery is more complex and overall results are worse if $\mathrm{OtoO}$ is present. ${ }^{6}$

Two of 3 patients had SSCD that explained surgical failure (the third remains unoperated). This probably occurred in our series because SSCD was not well recognized as a cause of failed otosclerosis surgery with persistent CHL until the past decade. SSCD may be present in $5 \%$ of patients presenting for surgical treatment of otosclerosis. ${ }^{17}$ SSCD acts as a "third window" mechanism, allowing dissipation of the mechanical fluid wave through an additional opening rather than transmission to the round window. ${ }^{18,19}$ Any labyrinthine dehiscence should be reported because a case of posterior semicircular canal dehiscence causing CHL is also reported. ${ }^{20-22}$

Progressive SNHL and/or vestibular symptoms occur in $0.6-3 \%$ of primary and up to $14 \%$ of revision stapes surgeries. ${ }^{23}$ Causes include serous or infectious labyrinthitis, labyrinthine fistula, reparative granuloma, and intravestibular foreign body. These are often less well evaluated with temporal bone CT, and enhanced MR imaging may be indicated. Three patients with sensorineural deafness and/or vestibular symptoms had CT findings that could explain their findings: 2 pistons with excessive vestibule penetration and 1 stapes footplate dislocation into the vestibule. Progressive cochlear otosclerosis in 2 patients may have contributed to SNHL on the basis of natural disease progression because most poststapedectomy patients have late development of SNHL (30 years out) in the course of their disease. ${ }^{24}$ Two patients also had labyrinthine ossificans as a suspected contributing cause of SNHL.

Limitations of this study include the occasionally incomplete nature of the electronic medical record and clinical correlation in 5 nonsurgical patients and 3 patients with cochlear implant surgery. There is also the possibility that pertinent intraoperative findings were not fully documented in the surgical reports. Furthermore, the prospective diagnostic capability of preoperative temporal bone CT was not assessed in this unblended, retrospective review. We were also unable to discriminate among patients with more than 1 potential contributing cause of persistent hearing and balance complaints.

\section{CONCLUSIONS}

Surgery is a mainstay of treatment for otosclerosis. Anatomic conditions that predispose to surgical failure include SSCD, OtoO, and labyrinthine ossificans. After initial surgery, temporal bone CT before revision surgery for persistent CHL may demonstrate prosthesis dislocation or disconnection, incus necrosis, granulation tissue, and inappropriate prosthesis size. SNHL and/or vestibular symptoms warrant assessment for suspected deep vestibular penetration or foreign body.

Disclosures: Bronwyn Hamilton—UNRELATED: Royalties: Amirsys, Comments: as above, expect less than $\$ 1000$ annually.

\section{REFERENCES}

1. Cummings CW, Fredrickson JM, Harker LA, et al, eds. Otolaryngology-Head and Neck Surgery. 2nd ed. St Louis: Mosby; 1993

2. Declau F, van Spaendonck M, Timmermans JP, et al. Prevalence of histologic otosclerosis: an unbiased temporal bone study in Caucasians. Adv Otorhinolaryngol 2007;65:6-16

3. Sakihara Y, Parving A. Clinical otosclerosis, prevalence estimates and spontaneous progress. Clin Otosc Prev Acta Otol 1999;199:468-72

4. Vincent R, Sperling, N, Oates, J, et al. Surgical findings and long-term hearing results in 3,050 stapedotomies for primary otosclerosis: a prospective study with the Otology-Neurotology Database. Otol Neurotol 2006;27(Suppl 2):S25-47 
5. Ozuer MZ, Olgun L, Gultekin G. Revision stapes surgery. Otolaryngol Head Neck Surg 2012;146:109-13

6. Williams MT, Ayache D, Elmaleh M, et al. Helical CT findings in patients who have undergone stapes surgery for otosclerosis. $A J R$ Am J Roentgenol 2000;174:387-92

7. Swartz JD, Lansman AK, Berger AS, et al. Stapes prosthesis: evaluation with CT. Radiology 1986;158:179-82

8. Moumoulidis I, Axon P, Baguley D, et al. A review on the genetics of otosclerosis. Clin Otolaryngol 2007;32:239-47

9. Curtin HD. Superior semicircular canal dehiscence syndrome and multi-detector row CT. Radiology 2003;226:312-14

10. Fatterpekar GM, Doshi AH, Dugar M, et al. Role of 3D CT in the evaluation of the temporal bone. Radiographics 2006;26(Suppl 1): S117-32

11. Bozzato A, Struffert T, Hertel V, et al. Analysis of the accuracy of high-resolution computed tomography techniques for the measurement of stapes prostheses. Eur Radiol 2010;20:566-71

12. Lesinski SG. Causes of conductive hearing loss after stapedectomy or stapedotomy: a prospective study of 279 consecutive surgical revisions. Otol Neurotol 2002;23:281-88

13. Warren FM, Riggs S, Wiggins RH. Computed tomographic imaging of stapes implants. Otol Neurotol 2008;29:586-92

14. Puxeddu R, Ledda GP, Pelagatti CL, et al. Revision stapes surgery for recurrent transmissional hearing loss after stapedectomy and stapedotomy for otosclerosis. Acta Otorhinolaryngol Ital 2005;25:347-52

15. Lagleyre S, Calmels MN, Escude B, et al. Revision stapes surgery: the "lateralized piston syndrome." Otol Neurotol 2009;30:1138-44
16. Choudhury N, Kumar G, Krishnan M, et al. Atypical incus necrosis: a case report and literature review. J Laryngol Otol 2008;122:1124-26

17. Picavet V, Govaere E, Forton G. Superior semicircular canal dehiscence: preview in a population with clinically suspected otosclerosis-type hearing loss. B-ENT 2009;5:83-88

18. Mikulec AA, McKenna MJ, Ramsey MJ, et al. CHL without vertigo: superior semicircular canal dehiscence presenting as conductive hearing loss without vertigo. Otol Neurotol 2004;25:121-29

19. Hope A, Fagan P. Latent superior canal dehiscence syndrome unmasked by stapedotomy for otosclerosis. J Laryngol Otol 2010;124: $428-30$

20. Gopen Q, Zhou G, Poe D, et al. Posterior semicircular canal dehiscence: first reported case series. Otol Neurotol 2012;33: $1368-74$

21. Merchant SN, Rosowski JJ, McKenna MJ. Superior semicircular canal dehiscence mimicking otosclerotic hearing loss. Adv Oto-RhinoLaryngol 2007;65:137-45

22. Nakashima T, Ueda H, Furuhashi A, et al. Air-bone gap and resonant frequency in large vestibular aqueduct syndrome. Am J Otol 2000;21:671-74

23. Yehudai, N, Luntz M. Resolution of delayed sudden sensorineural hearing loss after stapedectomy: a case report and review of the literature. Med J Otol 2006;3:156-60

24. Redfors YD, Moller C. Otosclerosis: thirty-year follow-up after surgery. Ann Otol Rhinol Laryngol 2011;120:608-14 